\title{
Labyrinthe
}

$9 \mid 2001$

Numéro 9

\section{La circulation des manuscrits du De excidio Troiae de Darès le Phrygien au Moyen Âge}

Louis Faivre d'Arcier

\section{(2) OpenEdition}

1 Journals

\section{Édition électronique}

URL : http://journals.openedition.org/labyrinthe/1123

DOI : $10.4000 /$ labyrinthe.1123

ISSN : 1950-6031

Éditeur

Hermann

\section{Édition imprimée}

Date de publication : 30 juin 2001

Pagination : 108-110

\section{Référence électronique}

Louis Faivre d'Arcier, "La circulation des manuscrits du De excidio Troiae de Darès le Phrygien au Moyen Âge », Labyrinthe [En ligne], 9 | 2001, mis en ligne le 11 février 2006, consulté le 21 avril 2019 URL : http://journals.openedition.org/labyrinthe/1123; DOI : 10.4000/labyrinthe.1123

Ce document a été généré automatiquement le 21 avril 2019.

Propriété intellectuelle 


\title{
La circulation des manuscrits du De excidio Troiae de Darès le Phrygien au Moyen Âge
}

\author{
Louis Faivre d'Arcier
}

1 Pourquoi se pencher sur la fortune d'un auteur aussi obscur que le pseudo-Darès le Phrygien*? Sans doute, parce qu'aucune étude sérieuse n'avait jamais été entreprise sur ce texte et en particulier sur sa très riche et abondante tradition manuscrite. Mais, en réalité, l'intérêt de ce texte comme source pour une histoire de la culture historique du Moyen Âge dépasse largement la valeur littéraire de cette courte œuvre latine. Écrite sans doute à la fin de l'Antiquité, dans la lignée d'un grand nombre de textes qui corrigeaient Homère et Virgile, cette version de la guerre de Troie est à la fois « rationaliste », au sens où les interventions divines sont supprimées, et d'un style très dépouillé. Pour ces raisons, elle a fait l'objet d'un certain mépris à partir de la fin $\mathrm{du} \mathrm{Xv}^{\mathrm{e}}$ siècle et jusqu'à une période très récente. L'objet de notre étude n'était pas de réhabiliter Darès (d'autres l'ont déjà fait). Il s'agissait plutôt de se pencher sur la tradition manuscrite du texte et de s'intéresser à la lecture de celui-ci au Moyen Âge.

2 Sachant qu'avant l'imprimerie, les textes étaient copiés de manuscrit en manuscrit, il peut être intéressant de raccorder les différents maillons de la chaîne en comparant le texte qu'ils contiennent. Les variantes que la comparaison - la collation - des manuscrits permet de repérer servent à former des familles. La comparaison des témoins doit permettre de restituer un texte aussi proche que possible de l'original, c'est-à-dire du texte tel que l'a voulu son auteur. Il serait un peu long de présenter tous les tenants et aboutissants du sujet, tant il s'est révélé complexe. On peut dire, néanmoins, qu'à l'issue de ce travail, qui s'est fondé à la fois sur les variantes du texte et sur l'histoire des manuscrits, la tradition manuscrite de Darès a pris un visage nouveau. En effet, à la suite de l'éditeur du siècle dernier, on considérait qu'il y avait deux familles (une bonne et une mauvaise, ce qui était assez commode). Or, les choses sont bien plus complexes. À côté de ces deux familles, l'existence d'une famille intermédiaire est avérée et, surtout, l'intérêt d'une version longue a été montré1. 
Ce travail a aussi permis d'apporter des éclairages sur l'histoire du texte et de sa réception. Le fait de repérer que deux témoins appartiennent à la même famille aide à comprendre l'histoire de la diffusion du texte. De plus, en repérant les textes avec lesquels Darès se trouvait associé dans les manuscrits médiévaux, il est possible de se rendre compte du contexte dans lequel les bibliothécaires et les lecteurs plaçaient Darès au Moyen Âge. De la sorte, on arrive à percevoir les raisons pour lesquelles on a tant copié ce texte, pourquoi on s'en est inspiré, pourquoi on l'a traduit dans les langues vernaculaires de toute l'Europe. De fait, plus de deux cents manuscrits nous sont parvenus, chiffre considérable, quand on sait que les Facta et dicta memorabilia de ValèreMaxime, œuvre historique la plus répandue au cours du Moyen Âge, sont représentés par environ quatre cent vingt témoins. Un tel succès, qui contraste avec le mépris actuel, ne peut que susciter les questions.

4 L'exemple de l'usage supposé de Darès dans les écoles paraît éclairant ${ }^{2}$. L'hypothèse scolaire était assez pratique : la forme succincte du récit, son apparent souci des faits, son peu d'intérêt pour les dieux de la mythologie s'y accordaient. Cette opinion, souvent énoncée comme une vérité établie depuis le XIX ${ }^{\mathrm{e}}$ siècle, s'appuyait sur quelques exemples. Darès est cité dans deux textes dus à la plume d'enseignants. Bernard Silvestre le mentionne comme plus véridique que Virgile dans son commentaire de l'Énéide, rédigé au début du XII ${ }^{\mathrm{e}}$ siècle. Au XIII ${ }^{\mathrm{e}}$ siècle, Évrard l'Allemand énumère dans son Laborintus, texte d'usage indubitablement scolaire, un certain nombre d'auteurs et de textes qui étaient lus ou pouvaient être lus dans les écoles de son temps, parmi lesquels figure Darès. Viennent en outre quelques considérations codicologiques ${ }^{3}$. En effet, Darès est associé avec Virgile très tôt dans la tradition manuscrite, alors que ce dernier est un des piliers du canon des auteurs scolaires. Le De excidio Troiae est aussi présent dans la partie scolaire de bibliothèques monastiques. En réalité, tous ces éléments ne constituent que des bribes de preuves.

5 La plus grande partie de notre documentation va dans un autre sens. Pas de commentaire, très peu de notes marginales dans les manuscrits. La place de Darès dans le manuscrit Paris, Bib. nat. de Fr., lat. 10307 est significative quant au statut de cet auteur et, sans doute aussi, quant à son usage ${ }^{4}$. Ce témoin est le plus scolaire d'aspect. J.-J. Contreni a vu dans le recueil la propriété d'un maitre lié à l'école de Laon, qui aurait vécu au $\mathrm{x}^{\mathrm{e}}$ siècle ${ }^{5}$. L'exemplaire de Darès qui y est inséré s'y trouve copié plus tard, à la fin du $\mathrm{x}^{\mathrm{e}}$ siècle, à l'instar d'une glose et du commentaire de Servius qui le précède, dans la marge extérieure du manuscrit. L'économie de la page montre bien qui a le dessus, du texte de l'énéide, copié d'une écriture d'assez grand module, ou de l'histoire, copiée dans une écriture de bien plus petite taille, à l'encre brune, presque jaune. Darès peut bien raconter la vérité de l'histoire, ce n'est pas à lui qu'appartient la première place. L'ensemble du manuscrit est assez mal conçu, puisque le texte du commentaire de Servius, qui semble avoir été écrit dès l'époque de la copie du Virgile, ne progresse pas au même rythme que le texte qu'il commente, si bien qu'il s'achève bien avant celui-ci. Par conséquent, la place de Darès est significative du statut qui lui est accordé, mais pas nécessairement de son usage : il est quasiment impossible, vu le poids du manuscrit, de passer aisément d'un texte à l'autre, d'autant plus que Darès se trouve bien loin du livre II de l'Énéide, celui qui raconte la chute de Troie, avec lequel la comparaison serait pertinente. Tous ces éléments incitent à penser que l'usage scolaire n'a jamais été qu'occasionnel et auxiliaire : c'étaient les faits rapportés par Darès qui comptaient. 
Or, la présence de Darès dans de gros recueils d'histoire ancienne permet de croire que si on lisait l'histoire de Troie, c'était pour se donner une teinture d'histoire universelle. En effet, le De excidio Troiae s'est trouvé fréquemment associé à l'histoire d'Alexandre racontée dans la tradition du pseudo-Callisthène ou à des histoires nationales, particulièrement celle des Anglais avec l'Historia regum Britanniae de Geoffroy de Monmouth. Le souci d'utiliser l'histoire de Darès comme un document pour l'histoire universelle se retrouve dans le découpage du texte en chapitres. Quoique assez variable d'un témoin à l'autre, la scansion des rubriques respecte la plupart du temps les mêmes principes, mettant en valeur les événements que constituent les combats devant Troie ${ }^{6}$ ainsi que les listes de personnages, nombreuses chez l'historien de la fin de l'Antiquité7. Plusieurs chroniques universelles médiévales se sont servies de notre texte. Or elles ont toutes retiré de Darès des dates et des chiffres, signe qu'on s'intéressait à lui avant tout pour les faits rapportés.

7 Le De excidio Troiae devait, à l'origine, être une sorte de jeu littéraire, créé pour un public de bons connaisseurs d'Homère et de Virgile : il s'est trouvé promu, au Moyen Âge, au rang de document historique. Il semble que seul un regard assez fin sur la tradition de l'œuvre permettait de percevoir cette métamorphose.

\section{NOTES}

*. Sont ici présentés les résultats d'une thèse de l'École des chartes, soutenue devant un jury composé de Pascale Bourgain, Jean Vezin, Françoise Vielliard et Robert Fossier. Ce travail est en cours de révision pour être publié.

1. Louis Faivre d'Arcier, « La circulation des manuscrits du De excidio Troiae de Darès le Phrygien ", Positions des thèses de l'École des chartes, Paris, 2000, p. 138-142.

2. Günther Glauche, Schullektüre im Mittelalter, Entstehung und Wandlungen des Lektürekanons bis 1200 nach den Quellen dargestellt, Munich, 1970. Birger Munk Olsen, I Classici nel canone scolastico altomedievale, Spolète, 1992, en particulier p. 93.

3. La codicologie peut être définie comme l'étude des manuscrits en tant qu'objets " archéologiques ", puisqu'elle cherche à en reconstituer l'origine et l'histoire.

4. Fac-similé de ce manuscrit dans : Antoinette Novara, "Virgile "latin" ", Mise en page et mise en texte du livre manuscrit, dir. Henri-Jean Martin et Jean Vezin, Paris, 1990, p. 145.

5. J.-J. Contreni, « À propos de quelques manuscrits de l'école de Laon au IX ${ }^{e}$ siècle : découvertes et problèmes ", Le Moyen Âge, 78, 1972, p. 31-38.

6. La plupart des manuscrits ont des rubriques du type " primum bellum », « bellum II » etc. 7. Les manuscrits, Paris, Bib. nat. de Fr., lat. 3359 et Londres, British Library, Royal 6 C. viii, constituent deux exemples très aboutis de rubrication du texte. 